\title{
Ancestral reconstruction of sunflower karyotypes reveals non-random chromosomal evolution
}

Kate L. Ostevik ${ }^{1,2}$, Kieran Samuk ${ }^{1}$, and Loren H. Rieseberg ${ }^{2}$

1. Department of Biology, Duke University, Durham, NC, 27701

2. Department of Botany, University of British Columbia, Vancouver, BC, Canada, V6T $1 Z 4$

Running Title: Chromosomal evolution in sunflower

Keywords: chromosomal rearrangement, synteny block, Helianthus, syntR, dot plot

Corresponding author:

Kate Ostevik

Box 90338, 137 Biological Sciences, 130 Science Drive, Durham, NC, 27708

984-227-0832

kate.ostevik@gmail.com 


\section{Abstract}

Mapping the chromosomal rearrangements between species can inform our understanding of genome evolution, reproductive isolation, and speciation. Here we present a novel algorithm for identifying regions of synteny in pairs of genetic maps, which is implemented in the accompanying R package, syntR. The syntR algorithm performs as well as previous ad-hoc methods while being systematic, repeatable, and is applicable to mapping chromosomal rearrangements in any group of species. In addition, we present a systematic survey of chromosomal rearrangements in the annual sunflowers, which is a group known for extreme karyotypic diversity. We build high-density genetic maps for two subspecies of the prairie sunflower, Helianthus petiolaris ssp. petiolaris and H. petiolaris ssp. fallax.

11 Using syntR, and we identify blocks of synteny between these two subspecies and previously published 12 high-density genetic maps. We reconstruct ancestral karyotypes for annual sunflowers using those 13 synteny blocks and conservatively estimate that there have been 7.9 chromosomal rearrangements 14 per million years - a high rate of chromosomal evolution. Although the rate of inversion is even higher 15 than the rate of translocation in this group, we further find that every extant karyotype is distinguished by between 1 and 3 translocations involving only 8 of the 17 chromosomes. This non-random exchange suggests that specific chromosomes are prone to translocation and may thus contribute disproportionately to widespread hybrid sterility in sunflowers. These data deepen our understanding of chromosome evolution and confirm that Helianthus has an exceptional rate of chromosomal rearrangement that may facilitate similarly rapid diversification.

\section{Introduction}

Organisms vary widely in the number and arrangement of their chromosomes - i.e., their karyotype.

24 Interestingly, karyotypic differences are often associated with species boundaries and, therefore, 25 suggest a link between chromosomal evolution and speciation (White 1978, King 1993). Indeed, it is well established that chromosomal rearrangements can contribute to reproductive isolation.

27 Individuals heterozygous for divergent karyotypes are often sterile or inviable (King 1987, Lai et al. 
2005, Stathos and Fishman 2014). Apart from directly causing hybrid sterility and inviability, chromosomal rearrangements can also facilitate the evolution of other reproductive barriers by extending genomic regions that are protected from introgression (Noor et al. 2001, Rieseberg 2001), accumulating genetic incompatibilities (Navarro and Barton 2003), and simplifying reinforcement (Trickett and Butlin 1994). Despite its prevalence and potentially important role in speciation, the general patterns of karyotypic divergence are still not well understood. Mapping and characterizing chromosomal rearrangements in many taxa is a critical step towards understanding their evolutionary dynamics.

The genus Helianthus (sunflowers) is well known to have particularly labile genome structure and is thus a viable system in which to map and characterize a variety of rearrangements. These sunflowers have several paleopolyploidy events in their evolutionary history (Barker et al. 2008, Barker et al. 2016, Badouin et al. 2017), have given rise to three homoploid hybrid species (Rieseberg 1991), and are prone to transposable element activity (Kawakami et al. 2011, Staton et al. 2012). Evidence in the form of hybrid pollen inviability, abnormal chromosome pairings during meiosis, and genetic map comparisons suggests that Helianthus karyotypes are unusually diverse (Heiser 1947, Heiser 1951, Heiser 1961, Whelan 1979, Chandler 1986, Rieseberg et al. 1995, Quillet et al. 1995, Burke et al. 2004, Heesacker et al. 2009, Barb et al. 2014). In fact, annual sunflowers have one of the highest described rates of chromosomal evolution across all plants and animals (Burke et al. 2004).

Studying chromosomal evolution within any group requires high-density genetic maps. Recently, Barb et al. (2014) built high-density genetic maps for the sunflower species $H$. niveus ssp. tephrodes and $H$. argophyllus and compared them to $H$. annuus. This analysis precisely mapped previously inferred karyotypes (Heiser 1951, Chandler 1986, Quillet et al. 1995), but only captured a small amount of the chromosomal variation in the annual sunflowers. For example, comparisons of genetic maps with limited marker density suggest that several chromosomal rearrangements differentiate $H$. petiolaris from $H$. annuus and (Rieseberg et al. 1995, Burke et al. 2004) and evidence from cytological surveys suggests that subspecies within H. petiolaris subspecies carry divergent karyotypes (Heiser 1961). 
us to: (1) precisely track additional rearrangements, (2) reconstruct ancestral karyotypes for the group, and (3) untangle overlapping rearrangements that can be obscured by directly comparing present-day

59 karyotypes.

61 Another critical part of a multi-species comparative study of chromosome evolution using genetic map data is a systematic and repeatable method for identifying syntenic chromosomal regions (sensu

63 Pevzner and Tesler 2003). These methods are especially important for cases with high marker density 64 because breakpoints between synteny blocks can be blurred by mapping error, micro-rearrangements, 65 and paralogy (Hackett and Broadfoot 2003, Choi et al. 2007, Barb et al. 2014, Bilton et al. 2018). In 66 previous studies, synteny blocks have been found by a variety of ad-hoc methods, including counting 67 all differences in marker order (Wu and Tanksley 2010), by visual inspection (Burke et al. 2004, Marone 68 et al. 2012, Latta et al. 2019), or by manually applying simple rules like size thresholds (Heesacker et al. 69 2009, Barb et al. 2014, Rueppell et al. 2016) and Spearman's rank comparisons (Berdan et al. 2014, 70 Schlautman et al. 2017). However, these methods become intractable and prone to error when applied 71 to very dense genetic maps. Furthermore, to our knowledge, there is no software available that 72 identifies synteny blocks based on relative marker positions alone (i.e., without requiring reference 73 genomes, sequence data, or markers with known orientations).

Here, with the goal of understanding chromosome evolution in Helianthus and more generally, we aimed to: (1) build high-density genetic maps for two subspecies of Helianthus petiolaris, (2) develop a method and software to systematically and repeatably identify synteny blocks from any number of paired genetic map positions, (3) reconstruct ancestral karyotypes for a subsection of annual sunflowers, and (4) detect general patterns of chromosomal rearrangement in Helianthus.

\section{Methods}

\section{Study system}


83 We focused on five closely related diploid $(2 n=34)$ taxa from the annual clade of the genus Helianthus

84 (Fig 1). These sunflowers are native to North America (Fig S1, Rogers et al. 1982) and are naturally self-

85 incompatible (domesticated lineages of $H$. annuus are self-compatible). Helianthus annuus occurs throughout much of the central United States, often in somewhat heavy soils and along roadsides (Heiser 1947). Helianthus petiolaris occurs in sandier soils and is made up of two subspecies: $H$. petiolaris ssp. petiolaris, which is commonly found in the southern Great Plains, and H. petiolaris ssp. fallax, which is limited to more arid regions in Colorado, Utah, New Mexico, and Arizona (Heiser 1961). Where $H$. petiolaris and $H$. annuus are sympatric, gene flow occurs between the species (Strasburg and Rieseberg 2008). Helianthus argophyllus is primarily found along the east coast of Texas where it also overlaps and hybridizes with $H$. annuus (Baute et al. 2016). Finally, H. niveus ssp. tephrodes is a facultative perennial that grows in dunes from the southwestern US into Mexico.

A

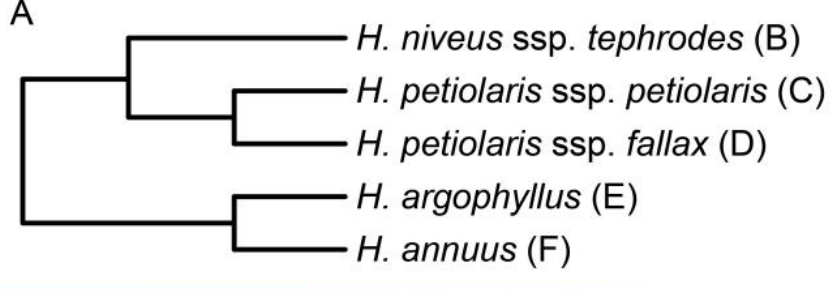

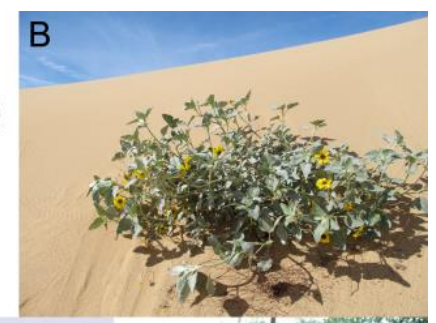

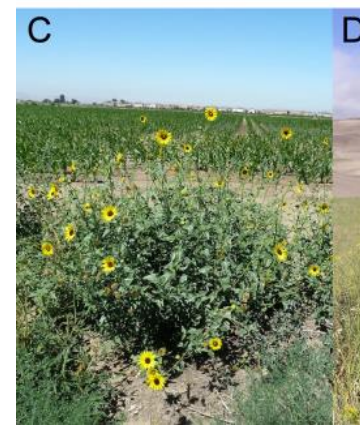

D
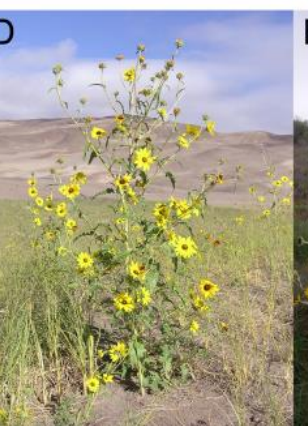

E

Figure 1 - The sunflower taxa used in this study. A) Phylogenetic relationships based on Stephens et al. (2015) and Baute et al. (2016). B) H. niveus ssp. tephrodes. C) H. petiolaris ssp. petiolaris. D) H. petiolaris ssp. fallax. E) H. argophyllus. F) H. annuus. Photo credits: Brook Moyers (B, C, E \& F) and Rose Andrew (D).

\section{Controlled crosses}

100 To make genetic maps, we crossed an outbred individual with presumably high heterozygosity from each $H$. petiolaris subspecies to a homozygous inbred line of domesticated sunflower and genotyped 
102 the resulting F1 offspring. This test-cross design allows us to infer where recombination occurred in the 103 heterozygous parents because we can reliably track the segregation of those parents' alleles against a 104 predictable background (Fig 2).
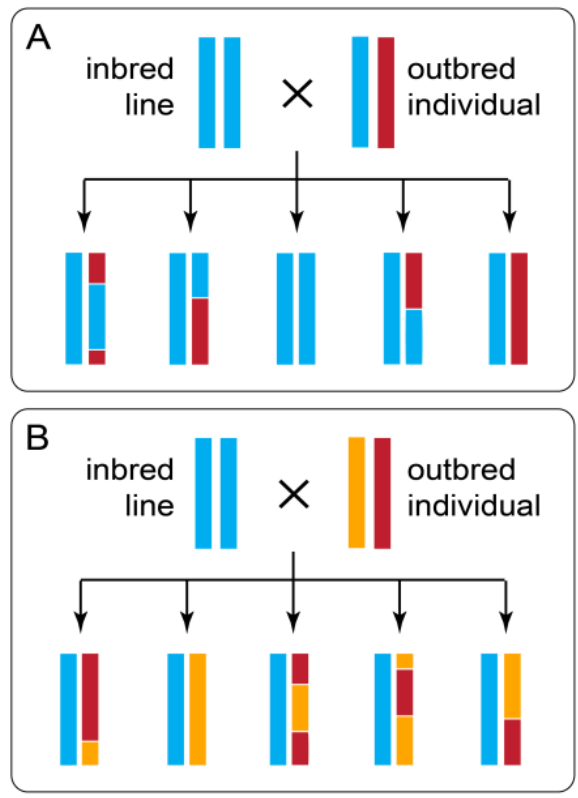

106 Figure 2 - Diagram showing how a test-cross can be used to map the recombination events in an outbred

107 individual that may (A) or may not (B) share alleles with the inbred line. Each line represents a chromosome, and 108 the colors represent ancestry.

110 Specifically, we used pollen from a single $H$. petiolaris ssp. petiolaris plant (PI435836) and a single $H$. 111 petiolaris ssp. fallax plant (PI435768) to fertilize individuals of a highly inbred and male sterile line of $H$. 112 annuus (HA89cms). The self-incompatible $H$. petiolaris accessions were collected in central Colorado 113 (PI435836, 39.741,$-105.342^{\circ}$, Boulder County) and the southeast corner of New Mexico (PI435768, $11432.3^{\circ},-104.0^{\circ}$, Eddy County, Fig S1) and were maintained at large population sizes by the United States 115 Department of Agriculture. When it was originally collected, accession PI435768 was classified $H$. 116 neglectus. However, based on the location of the collection (Heiser 1961) and a more recent genetic 117 analysis of the scale of differences between $H$. petiolaris ssp. fallax and $H$. neglectus (Raduski et al. 118 2010), we believe that this accession should be classified $H$. petiolaris ssp. fallax. 
121 We collected leaf tissue from $116 \mathrm{H}$. annuus $\times \mathrm{H}$. petiolaris ssp. petiolaris F1 seedlings and $132 \mathrm{H}$.

122 annuus $\times$ H. petiolaris ssp. fallax F1 seedlings. We extracted DNA using a modified CTAB protocol 123 (Doyle and Doyle 1987) and prepared individually barcoded genotyping-by-sequencing (GBS) libraries 124 using a version the Poland et al. (2012) protocol. Our modified protocol includes steps to reduce the 125 frequency of high-copy fragments (e.g., chloroplast and repetitive sequence) based on Shagina et al. 126 (2010) and Matvienko et al. (2013) and steps to select specific fragment sizes for sequencing (see 127 Ostevik 2016 appendix B for the full protocol).

Briefly, we digested 100ng of DNA from each individual with restriction enzymes (either Pstl-HF or Pstl$\mathrm{HF}$ and $\mathrm{Mspl}$ ) and ligated individual barcodes and common adapters to the digested DNA. We pooled barcoded fragments from up to 192 individuals, cleaned and concentrated the libraries using SeraMag Speed Beads made in-house (Rohland and Reich 2012), and amplified fragments using 12 cycles of PCR. We depleted high-copy fragments based on Todesco et al. (2019) using the following steps: (1)

134 denature the libraries using high temperatures, (2) allow the fragments to re-hybridize, (3) digest the 135 double-stranded fragments with duplex specific nuclease (Zhulidov et al. 2004), and (4) amplify the 136 undigested fragments using another 12 cycles of PCR. We ran the libraries out on a 1.5\% agarose gel and extracted 300-800 bp fragments using a Zymoclean Gel DNA Recovery kit (Zymo Research, Irvine, USA). Then, following additional library cleanup and quality assessment, we sequenced paired-ends of

141 To call variants, we used a pipeline that combines the Burrows-Wheeler Aligner version 0.7.15 (BWA, Li 142 \& Durbin 2010) and the Genome Analysis Toolkit version 3.7 (GATK, McKenna et al. 2010). First, we 143 demultiplexed the data using sabre (https://github.com/najoshi/sabre, Accessed 27 Jan 2017). Next, 144 we aligned reads to the $H$. annuus reference (HanXRQr1.0-20151230, Badouin et al. 2017) with 'bwa145 mem' (Li 2013), called variants with GATK 'HaplotypeCaller', and jointly genotyped all samples within a 146 cross type with GATK 'GentypeGVCFs'. We split variants into SNPs and indels and filtered each marker 147 type using hard-filtration criteria suggested in the GATK best practices (DePristo et al. 2011, Van der 
148 Auwera et al. 2013). Specifically, we removed SNPs that had quality by depth scores (QD) less than 2,

149 strand bias scores (FS) greater than 60, mean mapping quality (MQ) less than 40, or allele mapping bias

150 scores (MQRankSum) less than -12.5 and indels that had QD $<2$ or FS $>200$. After further filtering

151 variants for biallelic and triallelic markers with genotype calls in at least $50 \%$ of individuals, we used

152 GATK 'VariantsToTable' to merge SNPs and indels into a single variant table for each cross type.

154 Finally, we converted our variant tables into AB format, such that the heterozygous parents contribute 155 ' $A$ ' and ' $B$ ' alleles to offspring, while the $H$. annuus parent contributes exclusively ' $A$ ' alleles. At biallelic 156 markers (Fig 2A), sites with two reference alleles became ' $A A^{\prime}$ ' and sites with the reference allele, and 157 the alternate allele became 'AB'. At triallelic markers (Fig 2B), sites with the reference allele and one 158 alternate allele became 'AA' and sites with the reference allele, and the other alternate allele became 159 ' $A B$ '. This method randomly assigns ' $A$ ' and ' $B$ ' alleles to the homologous chromosomes in each 160 heterozygous parent, so our genetic maps initially consisted of pairs of mirror-imaged linkage groups 161 that we later merged.

\section{Genetic mapping}

We used R/qtl (Broman et al. 2003) in conjunction with R/ASMap (Taylor and Butler 2017) to build genetic maps. After excluding markers with less than $20 \%$ or greater than $80 \%$ heterozygosity and individuals with less than $50 \%$ of markers scored, we used the function 'mstmap.cross' with a stringent significance threshold $\left(p . v a l u e=1 \times 10^{-16}\right)$ to form conservative linkage groups. We used the function 'plotRF' to identify pairs of linkage groups with unusually high recombination fractions and the function 'switchAlleles' to reverse the genotype scores of one linkage group in each mirrored pair. We did this

172 Using the corrected genotypes, we made new linkage groups with only the most reliable markers.

173 Namely, we used the function 'mstmap.cross' (with the parameter values: dist.fun = "kosambi", p.value $174=1 \times 10^{-6}$, noMap.size $=2$, noMap.dist $=5$ ) on markers with less than $10 \%$ missing data and without 175 significant segregation distortion. We refined the resulting linkage groups by removing (1) markers 
176 with more than three double crossovers, (2) markers with aberrant segregation patterns (segregation

177 distortion more than two standard deviations above or below the mean segregation distortion of the 178 nearest 20 markers), and (3) linkage groups made up of fewer than four markers.

180 We progressively pushed markers with increasing amounts of segregation distortion and missing data 181 into the maps using the function 'pushCross'. After adding each batch of markers, we reordered the 182 linkage groups and dropped markers and linkage groups as described above. Once all the markers had 183 been pushed back, we used the function 'calc.errorlod' to identify possible genotyping errors (error 184 scores greater than 2) and replaced those genotypes with missing data. We continued to drop linkage 185 groups, markers, and genotypes that did not meet our criteria until none remained.

Finally, we dropped five excess linkage groups, each made up of fewer than 30 markers, from each map. The markers in these linkage groups mapped to regions of the $H$. annuus genome that were otherwise represented in the final genetic maps but could not be explained by reversed genotypes.

190 Instead, these markers were likely polymorphic in the HA89cms individual used for crosses because of 191 the 2-4\% residual heterozygosity in sunflower inbred lines (Mandel et al. 2013).

\section{SyntR development}

194 To aid in the identification of chromosomal rearrangements, we developed the R package 'syntR' 195 (code and documentation available at http://ksamuk.github.io/syntR). This package implements a 196 heuristic algorithm for systematically detecting synteny blocks from marker positions in two genetic 197 maps. The key innovation of the syntR algorithm is coupling a biologically-informed noise reduction 198 method with a cluster identification method better suited for detecting linear (as opposed to circular) 199 clusters of data points.

201 We based the syntR algorithm on the following statistical and biological properties of genetic maps and 202 chromosomal rearrangements: 
(1) Synteny blocks appear as contiguous sets of orthologous markers in the same or reversed order in pairs of genetic maps (Pevzner and Tesler 2003, Choi et al. 2007).

(2) The inferred order of markers in individual genetic maps is subject to error due to genotyping errors and missing data (Hackett and Broadfoot 2003). This error manifests as slight differences in the order of nearby markers within a linkage group between maps. This mapping error (which we denote 'error rate one') results in uncertainty in the sequence of markers in synteny blocks.

(3) In genomes with a history of duplication, seemingly orthologous markers can truly represent paralogs. These errors ('error rate two') look like tiny translocations and also disrupt marker orders within synteny blocks.

(4) When comparing genetic maps derived from genomes without duplications or deletions, every

223 The first step of the syntR algorithm is to smooth over mapping error (error rate one) by identifying 224 highly localized clusters of markers based on a genetic distance threshold (cM) in both maps using hierarchical clustering (Fig 3a). The number of clusters formed is determined by the parameter maximum cluster range $\left(\mathrm{CR}_{\max }\right)$ that defines the maximum genetic distance $(\mathrm{cM})$ that any cluster can span in either genetic map. After determining these initial clusters, we smooth the maps by collapsing each multi-marker cluster down into a single representative point (the centroid of the cluster) for processing in subsequent steps. Next, we address errors introduced by poorly mapped or paralogous markers (error rate two) by flagging and removing outlier clusters that do not have a neighboring 
231 cluster within a specified maximum genetic distance (cM), a parameter we denote nearest neighbor 232 distance ( $\mathrm{NN}_{\text {dist }}$, Fig $\left.3 \mathrm{~b}\right)$.

A) Initial cluster assignments
B) Outlier clusters

flagged for removal
C) Final synteny

block assignments
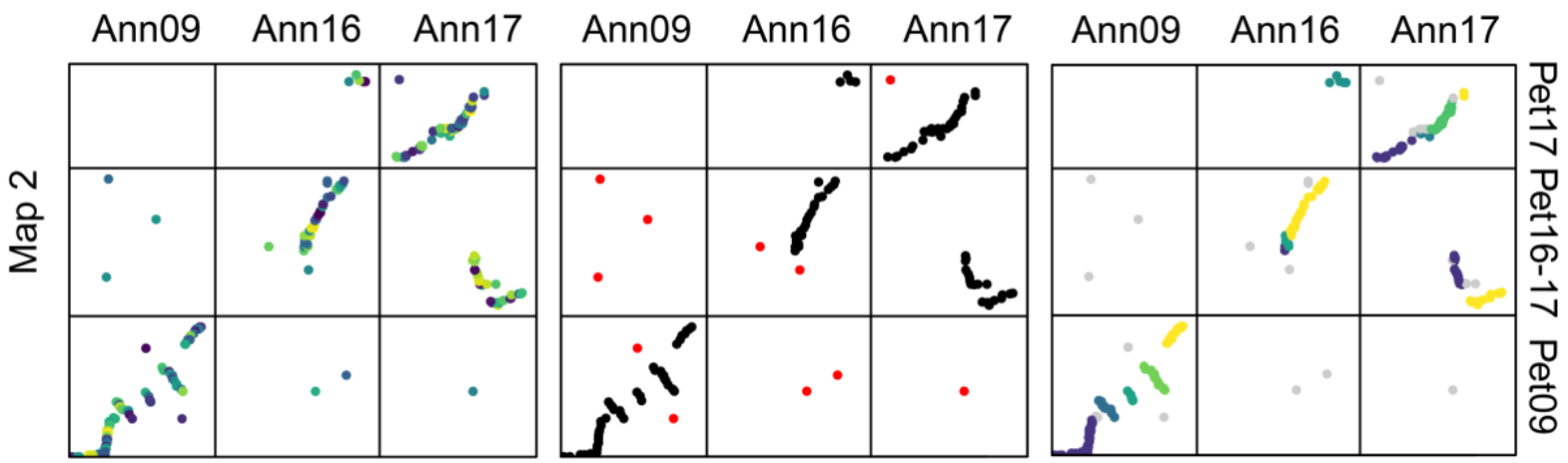

Map 1

Figure 3 - The stages of the syntR algorithm. Each plot shows the relationship between markers or clusters of markers from three chromosomes in two genetic maps. A) Highly localized markers are clustered. Each shade represents an individual cluster of markers that will be collapsed into a single representative point. B) Clusters without another cluster nearby are dropped. Red points represent clusters without a neighbor within $10 \mathrm{cM}$. C) Clusters are grouped into synteny blocks based on their rank positions. Grey points represent markers that were dropped in previous steps, and each other color represents a different synteny block.

After the noise reduction steps, we define preliminary synteny blocks using a method similar to the "friends-of-friends" clustering algorithm (Huchra and Geller 1982). First, we transform the genetic position of each cluster into rank order to minimize the impact of gaps between markers. We then group clusters that are (1) adjacent in rank position in one of the maps and (2) within two rank positions in the other map (Fig S2). This grouping method further reduces the effect of mapping error by aggregating over pairs (but not triplets) of clusters that have reversed orientations. If a minimum number of clusters per synteny block has been (optionally) defined, we sequentially eliminate blocks that fall below the minimum number of clusters, starting with blocks made up of one cluster and ending with blocks made up of clusters equal to one less than the minimum. After each elimination, we regroup the clusters into new synteny blocks. Finally, we adjust the extents of each synteny block by removing overlapping sections from both synteny blocks so that every position in each genetic map is uniquely represented (Fig 3c). 


\section{Assessing the performance of the syntR algorithm}

To evaluate the performance of this method and explore the effect of parameter choice on outcomes, we simulated genetic map comparisons with known inversion breakpoints and error rates in R. The genetic map comparisons were made by randomly placing 200 of markers at 100 positions along a 100 cM chromosome in two maps, reversing marker positions within a defined inversion region in one map, and then repositioning markers based on simulated mapping noise using the following two error parameters: (1) $E_{1}$ is the standard deviation of a normal distribution used to pick the distances markers are pushed out of their correct positions (e.g., when $\mathrm{ER}_{1}$ is $1 \mathrm{cM} \mathrm{95 \%} \mathrm{of} \mathrm{markers} \mathrm{will} \mathrm{be} \mathrm{within}$ $2 \mathrm{cM}$ of their true position); (2) $\mathrm{ER}_{2}$ is the proportion of markers that are repositioned according to a uniform distribution (i.e., these markers can be moved to any position on the simulated chromosome).

We initially ran syntR using fixed syntR parameters $\left(C R_{\max }=2\right.$ and $\left.N N_{\text {dist }}=10\right)$ on multiple simulated maps, which were made using variable parameters (inversion size: 2.5-50 cM, ER 1 : 0-2.0 cM, and ER 0-20\%), and counted the number of times the known breakpoints were identified within $1 \mathrm{cM}$ (Fig S3). As expected, we find that rearrangement size affects the false negative rate (i.e., failing to detect known breakpoints), such that smaller inversions are more likely to be missed (Fig S3c), but does not

271 affect the false positive rate (i.e., detecting breakpoints where there are none). We also find that increasing both types of error in the genetic maps tends to increase both the false positive and false negative rates, although $\mathrm{ER}_{1}$ has a much stronger effect on the false positive rate than any other

Using the same simulation methods as above but now varying the syntR parameter $C R_{\max }$, we find that small values of $\mathrm{CR}_{\max }$ yield high false positive rates while large values yield high false negative rates (Fig S4a). In addition, the $\mathrm{ER}_{1}$ parameter has a strong effect on the relationship between $\mathrm{CR}_{\max }$ and the false positive rate. Higher values of $\mathrm{CR}_{\max }$ are needed to reduce the false positive rate when $\mathrm{ER}_{1}$ is also high

(Fig S4b). This means that picking an appropriate $\mathrm{CR}_{\max }$ value is key to the accuracy of this method. 
284 When the syntR heuristic algorithm is performing well, the final synteny blocks should represent all 285 positions in the two genetic maps being compared (Chen et al. 2009). Based on this characteristic, we developed a method to choose optimal syntR tuning parameters ( $C R_{\max }$ and $\left.\mathrm{NN}_{\text {dist }}\right)$ that maximize the representation of the genetic maps and markers in synteny blocks. In this method a user: (1) runs syntR with a range of parameter combinations; (2) saves summary statistics about the genetic distance of each map represented in the synteny blocks and the number of markers retained for each run; and (3) finds the parameter combination that maximizes a composite statistic that equally weights these three measures. In cases where there are multiple local maxima, we suggest choosing the local maximum with the smallest value of $\mathrm{CR}_{\max }$ to reduce the number of potential false positives.

The "maximize representation" method for choosing syntR parameters has several benefits. First, it does not rely on any additional information (e.g., error rate estimates from the genetic maps compared). Second, when we use this method to choose the best parameters for simulated genetic maps, we find that these parameter values also minimize false positive and false negative rates (Fig S5). Third, when we simulate biologically realistic genetic map comparisons, the absolute value of false positives and false negatives are small. For example, when comparing two genetic maps in which 95\% of markers are within $1 \mathrm{cM}$ of their true position $\left(\mathrm{ER}_{1}=0.5\right)$ and $5 \%$ of markers are randomly permuted $301\left(\mathrm{ER}_{2}=0.05\right)$, nonexistent breakpoints will be identified 0.1 times and a breakpoint of a $20 \mathrm{cM}$ inversion 302 will be missed 0.04 times. These low error rates also highlight the overall robustness and accuracy of 303 the syntR algorithm.

In addition to performing simulations, we compared the synteny blocks identified by syntR to those identified by other means in a previously published comparison of $H$. niveus ssp. tephrodes and $H$. 308 input into syntR and used the "maximize representation" method to determine the optimal parameter 309 values for the two comparisons ( $H$. niveus vs. $H$. annuus: $\mathrm{CR}_{\max }=1.5, \mathrm{NN}_{\text {dist }}=30 ; H$. argophyllus vs. $H$. 310 annuus: $\mathrm{CR}_{\max }=2, \mathrm{NN}_{\text {dist }}=20$ ). We found that syntR was in strong agreement with previous work (Fig 311 S6), recovering all the same translocations and most of the same inversions as the Barb et al. (2014) 
312 maps. Most of the cases of mismatches were very small or weakly supported inversions in the Barb et

313 al. (2014) maps that syntR did not identify.

315 Finding synteny blocks

317 We used syntR to identify synteny blocks between our newly generated genetic maps and an ultra-

318 high-density map of $H$. annuus that was used to build the sunflower genome that we use as a reference

319 (Badouin et al. 2017). This allowed us to easily convert between physical position in the $H$. annuus

320 reference and position in the $H$. annuus genetic map. Using this property, we further compared two

321 previously published genetic maps for the closely related sunflower species, $H$. niveus ssp. tephrodes

322 and $H$. argophyllus (Barb et al. 2014), to the same $H$. annuus map. We aligned marker sequences from

323 the published maps to the $H$. annuus reference using bwa and converted well-aligned markers (MQ >

324 40) to their positions in the $H$. annuus genetic map.

Initially, we ran syntR using parameters identified through the "maximize representation" method for each map comparison separately (Table S1). However, varying $\mathrm{CR}_{\max }$ revealed rearrangements that were shared between the maps (Fig S7). Therefore, we ran syntR again using a range of $\mathrm{CR}_{\max }$ values that included the best fit for each comparison (1.0 - 3.5 in 0.5 increments) and extracted a curated set of synteny blocks from the output. A synteny block was retained if it fulfilled any of the following criteria (in decreasing order of importance): (1) it was found in another species, (2) it was identified in the majority of syntR runs for a single species, (3) it maximized the genetic distance represented by synteny blocks. We present this curated set of synteny blocks below, but our results are unchanged if

336 We named the chromosomes in our genetic maps based on their synteny with the standard order and 337 orientation of $H$. annuus chromosomes (Tang et al. 2002, Bowers et al. 2012) following Barb et al.

338 (2014) but with shortened prefixes $(A=H$. annuus, $R=H$. argophyllus, $N=H$. niveus ssp. tephrodes, $P=$ 
H. petiolaris ssp. petiolaris, $\mathrm{F}=H$. petiolaris ssp. fallax). For example, an $H$. petiolaris ssp. fallax

chromosome made up of regions that are syntenic with $H$. annuus chromosomes 4 and 7 is called F4-7.

344 We used our inferred synteny blocks and the software MGR v 2.01 (Bourque and Pevzner 2002) to infer

345 ancestral karyotypes for our five Helianthus taxa and to determine the number of chromosomal

346 rearrangements that occurred along each branch of the species tree. To run the MGR analysis, we

347 needed the order and orientations of synteny blocks in all five maps. However, individual synteny

348 blocks were often missing from one or more of our final maps. We approached this problem in two

349 ways. First, we inferred the likely position of missing synteny blocks based on the location of markers

350 that were too sparse to be grouped by syntR and matched the location of synteny blocks in other

351 maps. In the second case, we dropped any synteny blocks that were not universally represented.

352 Because we already had two sets of synteny blocks for each map (curated and individually optimized),

353 we ran the MGR analyses using three different sets of synteny blocks: (set 1) curated and inferred, (set

354 2) curated and present in all five maps, (set 3) individually optimized and present in all five maps.

358 The R program, syntR, is available on GitHub: https://github.com/ksamuk/syntR. The sequences used 359 to generate genetic maps are available on the SRA: http://www.ncbi.nlm.nih.gov/bioproject/598366.

360 All other data and scripts are available on dryad: https://doi.org/10.5061/dryad.7sqv9s4pc.

\section{Results}

\section{Genetic maps}


364 Both $H$. petiolaris genetic maps are made up of the expected 17 chromosomes and have very high

365 marker density (Fig 4, Fig S8). Only 6\% of the $H$. petiolaris ssp. petiolaris map and $10 \%$ of the $H$.

366 petiolaris ssp. fallax map fails to have a marker within 2 cM (Fig S9). Overall, both maps are somewhat

367 longer than the H. petiolaris map reported by Burke et al. (2004). Although this could represent real

368 variation between genotypes, it could also be the result of spurious crossovers that are inferred based

369 on genotyping errors. Because genotyping errors are proportional to the number of markers, maps

370 with high marker densities are more likely to be inflated. Indeed, building maps with variants that were

371 thinned to 1 per 150 bp using vcftools version 0.1 .13 (Danecek et al. 2011) yields collinear maps that

372 are closer to the expected lengths (Table S2, Fig S10). We present subsequent results based on the full

373 maps to improve our resolution for detecting small rearrangements.

375 Despite the general expansion of our maps, we find that chromosomes 2 and 4 in the H. petiolaris ssp. 376 fallax map (F2 and F4) are unexpectedly short (Fig 4). When we look at the distribution of markers for 377 this map relative to the $H$. annuus reference, we find very few variable sites in the distal half of these 378 chromosomes (Fig S11). That is, this individual was homozygous along vast stretches of F2 and F4.

379 These runs of homozygosity could be explained by recent common ancestry (i.e., inbreeding) or a lack 380 of variation in the population (e.g, because of background selection or a recent selective sweep).

381 Regardless, the lack of variable sites within the $H$. petiolaris ssp. fallax individual used for crosses 382 explains the shortness of F2 and F4. Notably, we find the same pattern on the distal half of $H$. annuus 383 chromosome 7 and find that this region is also not represented in the $H$. petiolaris spp. fallax map. 

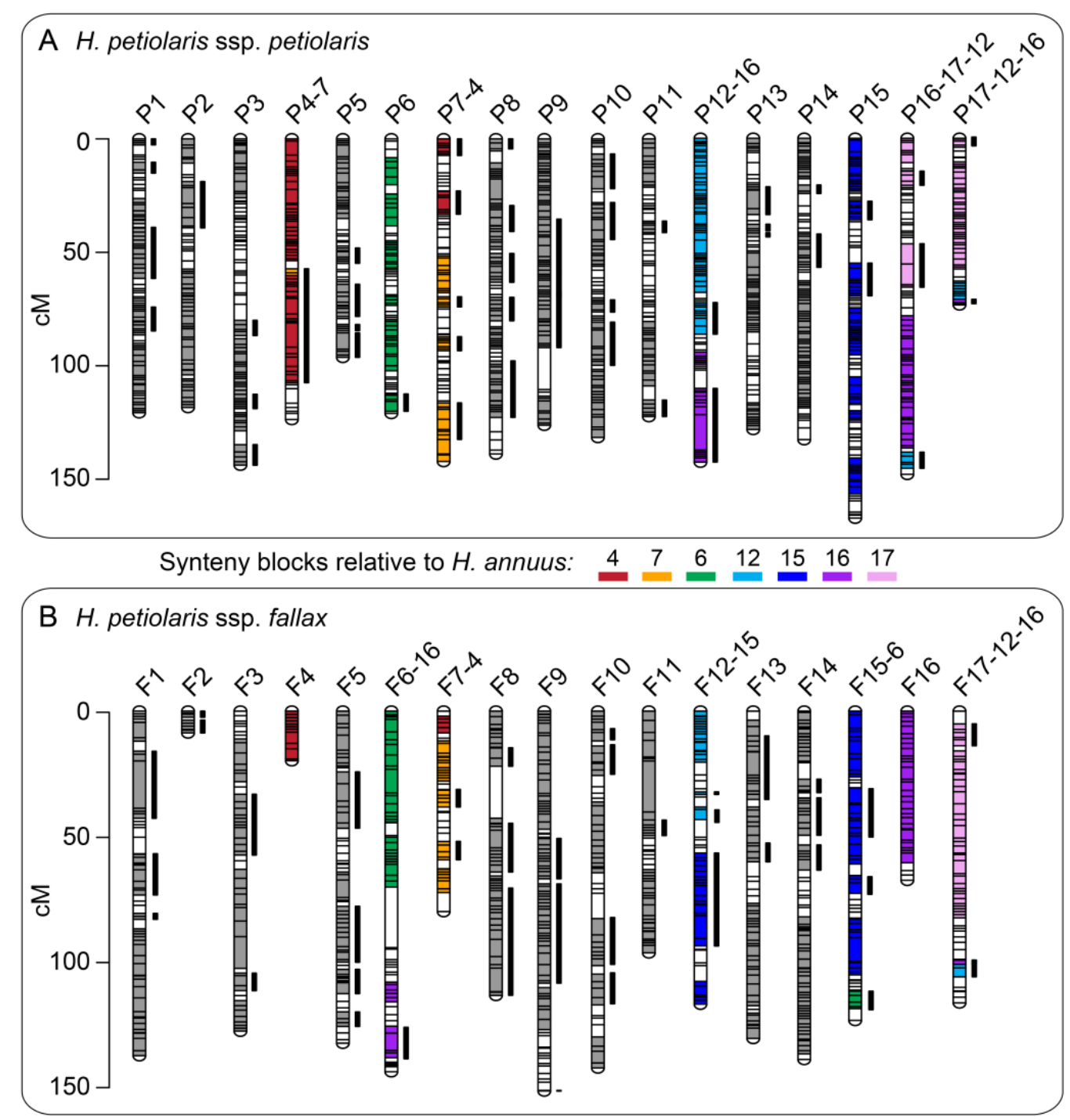

385 Figure 4 - Helianthus petiolaris genetic maps showing blocks of synteny with $H$. annuus. Each horizontal bar

386 represents a genetic marker. The thick vertical bars next to chromosomes represent synteny blocks that are

387 inverted relative to the $H$. annuus genetic map. Where there are no translocations between $H$. petiolaris and $H$.

388 annuus chromosomes (e.g.. all synteny blocks in P1 and F1 are syntenic with A1), the synteny blocks are shown

389 in grey. Where there are translocations, the synteny blocks are color-coded based on their synteny with $H$.

390 annuus chromosomes. Regions that are not assigned to a synteny block remain white. The synteny blocks

391 plotted are those curated based on multiple runs of syntR using different parameters. Please see Fig S12 for a

392 labeled version. This figure was made with LinkageMapView (Ouellette et al. 2017). 


\section{Synteny blocks}

Using syntR, we recovered 97 genetic regions that are syntenic between the $H$. petiolaris ssp. petiolaris and $H$. annuus and 79 genetic regions that are syntenic between the $H$. petiolaris ssp. fallax and $H$. annuus (Fig 4). We also recovered synteny blocks for the $H$. niveus ssp. tephrodes and $H$. argophyllus comparisons that are similar to those found previously (Fig S13). In all four comparisons, syntR successfully identified synteny blocks that cover large proportions (63\%-90\%) of each genetic map even in the face of a very high proportion of markers that map to a different chromosome than their neighbors (Table 1). These "rogue markers" could be the result of very small translocations, poorly mapped markers, or extensive paralogy. Over and above the prevalence of rogue markers, the karyotypes we recovered are substantially rearranged. Only between $32 \%$ and $45 \%$ of synteny blocks

Table 1 - Properties of the synteny blocks found using a syntR analysis between genetic maps of $H$. annuus and four other Helianthus taxa. The proportion of rogue markers is based only on the chromosomes without translocations in any map (i.e., chromosomes 1-3, 5, 8-10, 11, and 14). For those chromosomes, the majority of marker mapped to a single $H$. annuus chromosome. The other markers are considered rogue.

\begin{tabular}{lccccccc}
\hline Genetic map & $\begin{array}{c}\text { N synteny } \\
\text { blocks }\end{array}$ & $\begin{array}{c}\text { Rogue } \\
\text { markers }\end{array}$ & $\begin{array}{c}\text { Map } \\
\text { coverage }\end{array}$ & $\begin{array}{c}\text { H. annuus } \\
\text { coverage }\end{array}$ & Collinear & Inverted & Translocated \\
\hline H. petiolaris ssp. petiolaris & 97 & $19 \%$ & $80 \%$ & $74 \%$ & $39 \%$ & $36 \%$ & $26 \%$ \\
H. petiolaris spp. fallax & 79 & $17 \%$ & $63 \%$ & $65 \%$ & $32 \%$ & $34 \%$ & $34 \%$ \\
H. niveus ssp. tephrodes & 43 & $26 \%$ & $78 \%$ & $75 \%$ & $40 \%$ & $21 \%$ & $39 \%$ \\
H. argophyllus & 31 & $20 \%$ & $90 \%$ & $82 \%$ & $45 \%$ & $16 \%$ & $39 \%$ \\
\hline
\end{tabular}
for each map are collinear with the $H$. annuus genetic map in direct comparisons (Table 1 ).

\section{Karyotype reconstruction and chromosomal rearrangement}

413 Because nested and shared rearrangements can obscure patterns of chromosome evolution, we use 414 the MGR analyses to predict the most likely sequence of rearrangements in a phylogenetic context 415 before quantifying the rearrangement rate. These MGR analyses identified similar patterns of chromosome evolution regardless of the exact set of synteny blocks that we used (Table S5). Multiple 
417 taxa share many rearrangements, and the similarity of karyotypes matches known phylogenetic

418 relationships. Moreover, MGR analyses run without a guide tree inferred the known species tree, and

419 MGR analyses run with all other topologies identified an inflated number of chromosomal

420 rearrangements.

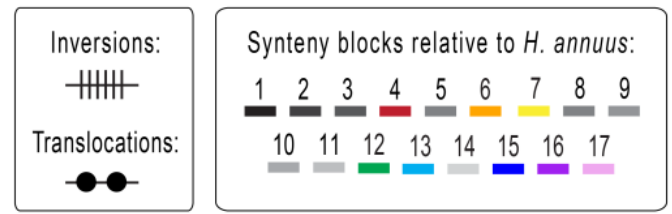

Figure 5 - Diagram showing the karyotypes of 5 Helianthus taxa as well as reconstructed ancestral karyotypes and the locations of chromosomal rearrangements. The karyotypes were built using synteny block set 1 , which were curated based on multiple syntR runs and inferred when missing. Each synteny block is represented using a line segment that is color-coded based on its position in the $H$. annuus genome (see Fig S14 for a labeled version). Chromosomes without translocations in any map are plotted in grey, and synteny blocks that are inverted relative to $H$. annuus are plotted using arrows. Also, note that along some branches the same pair of

Using the most complete set of synteny blocks (set 1), we find that 88 chromosomal rearrangements occurred across the phylogeny (Fig 5). Then, using the most current divergence time estimates for this group (Todesco et al. 2019) and conservatively assuming that $H$. niveus ssp. tephrodes diverged at the earliest possible point, we estimate that 7.9 (7.8-8) rearrangements occurred per million years in this clade (Tables S3-S5). To further explore the potential range of rearrangement rates, we considered 
436 other estimates of divergence times in sunflower (Sambatti et al. 2012, Mason 2018) and the other

437 sets of synteny blocks. Overall, the lowest rate we identified was 2.6 rearrangements per million years,

438 while the highest rate was indeterminable because some minimum divergence time estimates for the

439 group include 0 (Tables S3-S5).

441 The 88 rearrangements include 74 inversions and 14 translocations that are quite evenly distributed 442 across the phylogeny. However, the excess inversions indicate that it is unlikely that the rate of 443 inversions is equal to the rate of translocation (binomial test, $5.1 \times 10^{-11}$ ). Furthermore, we find that only 4448 of the 17 chromosomes are involved in the 14 translocations we identified. If translocations were 445 equally likely for all chromosomes, this asymmetry is very unlikely to have happened by chance (the 446 probability of sampling $\leq 8$ chromosomes in 14 translocations is $8.0 \times 10^{-8}$, Fig S15), suggesting that 447 some chromosomes are more likely to be involved in translocations than other. In line with this 448 observation, we see that some chromosome segments are repeatedly translocated. For example, A4 449 and $A 7$ are involved in several exchanges, and part of A6 has a different position in almost every map 450 (Fig 5). 


\section{Discussion}

452 Large-scale chromosomal changes may be key contributors to the process of adaptation and 453 speciation, yet we still have a poor understanding of rates of chromosomal rearrangement and the 454 evolutionary forces underlying those rates. Here, we devised a novel, systematic method for 455 comparing any pair of genetic maps, and performed a comprehensive analysis of the evolution of 456 chromosomal rearrangements in a clade of sunflowers. We created two new genetic maps for 457 Helianthus species and used our new method to identify a wide range of karyotypic variation in our 458 new maps, as well as previously published maps. Consistent with previous studies, we discovered a 459 high rate of chromosomal evolution in the annual sunflowers. Further, we found that inversions are 460 more common than translocations and that certain chromosomes are more likely to be translocated. 461 Below, we discuss the evolutionary and methodological implications of this work and suggest some 462 next steps in understanding the dynamic process of chromosomal rearrangement.

Studying the evolution of chromosomal rearrangements requires dense genetic maps and systematic methods to analyze and compare these maps between species. Our new software, syntR, provides an end-to-end solution for systematic and repeatable identification of synteny blocks in pairs of genetic maps with any marker density. Our tests on real and simulated data find that syntR recovers chromosomal rearrangements identified previous by both manual comparisons and cytological study,

472 Overall, we believe syntR will be a valuable tool for the systematic study of chromosomal 473 rearrangements in any species. The only data syntR needs to identify synteny blocks is relative marker 474 positions in two genetic maps. This fact is significant because, although the number of species with 475 whole genome sequence and methods to detect synteny blocks from those sequences are rapidly 476 accumulating, such as Mauve (Darling et al. 2004), Cinteny (Sinha and Meller 2007), syMAP (Soderlund 477 et al. 2011), SynChro (Drillion et al. 2014) and SyRI (Goel et al. 2019), it is still uncommon to have 
multiple closely related whole genome sequences that are of sufficient quality to compare for

karyotype differences. At the same time, the proliferation of reduced representation genome sequencing methods means that it is easy to generate many genetic markers for non-model species and produce very dense genetic maps. Furthermore, syntR allows comparisons to include older genetic map data that would otherwise go unused. The simplicity of the syntR algorithm will facilitate rapid karyotype mapping in a wide range of taxa.

We also believe that syntR provides a baseline for the development of further computational and statistical methods for the study of chromosomal rearrangements. One fruitful direction would be to integrate the syntR algorithm for synteny block detection directly into the genetic map building process (much like GOOGA, Flagel et al. 2019). Another key extension would be to allow syntR to compare multiple genetic maps simultaneously to detect synteny blocks in a group of species (e.g., by leveraging information across species). Finally, formal statistical methods for evaluating the model fit

491 and the uncertainty involved with any set of synteny blocks would be a major (albeit challenging)

492 improvement to all existing methods, including syntR.

\section{The similarity of $H$. petiolaris maps to previous studies}

Compared with previous work, we found more inversions and fewer translocations between $H$.

petiolaris subspecies and $H$. annuus (Rieseberg et al. 1995, Burke et al. 2004). This is probably due to a combination of factors. First, there appears to be karyotypic variation within some Helianthus species (Heiser 1948, Heiser 1961, Chandler et al. 1986). Second, the maps presented here are made up of more markers and individuals, which allowed us to identify small inversions that were previously undetected as well as to eliminate false linkages that can be problematic in small mapping populations. Lastly, we required more evidence to call rearrangements. Although we recovered some of the translocations supported by multiple markers in Rieseberg et al. (1995) and Burke et al. (2004), we did not recover any of the translocations supported by only a single sequence-based marker. Given the high proportion of "rogue markers" in our maps, it is likely that some of the putative translocations recovered in those earlier comparisons are the result of the same phenomenon. 
On the other hand, we found that rearrangements between our H. petiolaris maps match the

508 translocations predicted from cytological studies quite well. Heiser (1961) predicted that $H$. petiolaris ssp. petiolaris and H. petiolaris ssp. fallax karyotypes would have three chromosomes involved in two translocations that form a ring during pairing at meiosis, as well as the possibility of a second

511 independent rearrangement. This exact configuration is likely to occur at meiosis in hybrids between

512 the H. petiolaris subspecies maps we present here (Fig S16). Also, the most noteworthy chromosome 513 configuration in cytological studies of H. annuus-H. petiolaris hybrids (Heiser 1947, Whelan 1979, 514 Ferriera 1980, Chandler et al. 1986) was a hexavalent (a six-chromosome structure) plus a quadrivalent

515 (a four-chromosome structure). Again, this is the configuration that we would expect in a hybrid 516 between $H$. annuus and the $H$. petiolaris ssp. petiolaris individual mapped here. Furthermore, the 517 complicated arrangement and relatively small size of A12, A16 and A17 synteny blocks in $\mathrm{H}$. petiolaris 518 might explain why cytological configurations in $H$. annuus-H. petiolaris hybrids are so variable.

519 Interestingly, the rearrangements identified between $H$. argophyllus and $H$. annuus karyotypes here 520 and in Barb et al. (2014) also match the cytological studies better than an earlier comparison of sparse 521 genetic maps (Heesacker et al. 2009). It seems that, in systems with the potential for high proportions 522 of rogue markers, many markers are needed to identify chromosomal rearrangements reliably.

Our data suggest that annual sunflowers experience approximately 7.9 chromosomal rearrangements per million years. This rate overlaps with recent estimates for this group (7.4-10.3, Barb et al. 2014) and is even higher than the estimate that highlighted sunflower as a group with exceptionally fast chromosomal evolution (5.5-7.3, Burke et al. 2004). However, since Burke et al. (2004), chromosomal rearrangements have been tracked in many additional groups, including mammals (Ferguson-Smith 531 and Trifonov 2007, Martinez et al. 2016, da Silva et al. 2019), fish (Molina et al. 2014, Ayres-Alves et al. 532 2017), insects (Rueppell et al. 2016, Corbett-Detig et al. 2019), fungi (Sun et al. 2017) and plants 533 (Yogeeswaran et al. 2005, Schranz et al. 2006, Huang et al. 2009, Vogel et al. 2010, Latta et al. 2019). Of 
534 these analyses, relatively few have systematically studied karyotypes evolution across multiple species and estimated total rearrangement rates. Of those that do, most studies report less than 7.9

536 chromosomal rearrangements per million years, for example, in Solanum (0.36-1.44, Wu and Tanksley

537 2010), Drosophila (0.44-2.74, Bhutkar et al. 2008) and mammals (0.05-2.76, Murphy et al. 2005). But

538 there are exceptions, such as a comparison of genome sequences that revealed up to 35.7

539 rearrangements per million years in some grass lineages (Dvorak et al. 2018).

541 At the same time, we are likely underestimating rearrangement rates here for two reasons. First, we 542 used conservative thresholds for calling rearrangements. For example, some proportion of the rogue 543 markers that we identified could be the result of very small but real chromosomal rearrangements. 544 Second, our ability to resolve very small synteny blocks and breakpoints between synteny blocks 545 depends on marker density. Until we have full genome sequences to compare (like for the grass 546 lineages), we could be failing to detect very small rearrangements and falsely inferring that 547 independent rearrangements are shared. However, regardless of just how much we are 548 underestimating the rate, sunflower chromosomes are evolving quickly. This high rate of chromosomal 549 evolution could be a consequence of a higher rate of chromosomal mutation, a decreased chance that 550 chromosomal polymorphisms are lost, or both processes.

\section{Type of rearrangements}

554 We found that inversions and interchromosomal translocations dominate chromosomal evolution in Helianthus. This pattern is common in angiosperm lineages (Weiss-Schneeweiss and Schneeweis 2012) and fits with the consistent chromosome counts across annual sunflowers $(2 n=34$, Chandler et al. 1986). In addition, we found more inversions than translocations, which has previously been seen in both plant (Wu and Tanksley 2010, Amores et al. 2014) and animal systems (Rueppell et al. 2016) and echoes general reports that intrachromosomal rearrangements are more common than interchromosomal rearrangements (Pevzner and Tesler 2003). These consistent rate differences are notable because, although both rearrangement types depend on double strand breaks, two of the 
562 major consequences of chromosomal rearrangements, underdominance (i.e., rearrangement

563 heterozygotes are less fit than either homozygote) and recombination modification, might be more

564 common for some types of rearrangements.

566 Translocations have a more predictable effect on hybrid fertility, while inversions consistently reduce

567 recombination. Reciprocal translocation heterozygotes can affect fertility because missegregation 568 during meiosis can cause half of the gametes to be unbalanced and thus inviable (White 1973, King

569 1993). Although inversion heterozygotes can also produce unbalanced gametes, whether that happens

570 is dependent on the size of the inversion and whether disrupted pairing during meiosis inhibits

571 crossovers (Searle 1993). When inversions are small or have suppressed crossing over, they will not be

572 strongly underdominant. On the other hand, inversions often exhibit reduced recombination either

573 because recombination is suppressed through disrupted pairing (Searle 1993) or ineffective through

574 the production of inviable gametes (Rieseberg 2001). While interactions between reduced

575 recombination and adaptation with gene flow have been extensively examined in the case of

576 inversions (Kirkpatrick and Barton 2006, Hoffman and Rieseberg 2008, Yeaman and Whitlock 2011,

577 Yeaman 2013), it is not clear whether the same pattern will be common for translocations (but see

578 Fishman et al. 2013, Stathos and Fishman 2014 for one example). Translocations bring together

579 previously unlinked alleles and mispairing at translocation breakpoints could suppress crossing over,

580 but recombination inside reciprocal translocations will not necessarily produce inviable gametes and

581 thus reduce effective recombination.

582

583 Although any selective force could be responsible for the evolution of any chromosomal

584 rearrangement, potential differences in the relative magnitude of underdominance versus

585 recombination suppression may contribute to the evolution of sunflower chromosomes. While many

586 chromosomal rearrangements in sunflowers appear to be strongly underdominant (Chandler 1986, Lai

587 et al. 2005), inversions typically are not (L. Rieseberg, unpublished). If translocations tend to be more

588 underdominant than inversions, they would be less likely to evolve through drift and more likely to

589 cause reproductive isolation directly. This could explain why translocations are less common than

590 inversions and why pollen viability is accurately predicted by the number of translocations inferred 
591 from cytological studies (Chandler et al. 1986). At the same time, recent genomic analyses have

592 identified several extensive regions of very low recombination caused by large inversions segregating

593 in natural sunflower populations (Todesco et al. 2019, Huang et al. 2019). Mutations that segregate for

594 extended periods are unlikely to be strongly underdominant, and these inversions are associated with

595 multiple adaptive alleles (Todesco et al. 2019), which is consistent with a role for selection in their

596 origin or maintenance.

Non-random chromosomal rearrangement

We also found that some sunflower chromosomes are involved in more translocations than others.

601 This pattern has been observed in wheat (Badaeva et al. 2007) and breakpoint reuse is a common

602 phenomenon in comparative studies of karyotypes (Pevzner and Tesler 2003, Bailey et al. 2004,

603 Murphy et al. 2005, Larkin et al. 2009). Many studies support the idea that chromosomal regions with

604 greater sequence similarity are more likely to recombine and thus potentially generate novel

605 chromosomal arrangements. Some of the clearest examples of this come from the polyploidy

606 literature, where chromosomes with ancestral homology are more likely to recombine (Nicolas et al.

607 2007, Marone et al. 2012, Mason et al. 2014, Tennessen et al. 2014, Nguepjop et al. 2016). However,

608 centromeres and other repetitive regions can also affect the rate of mutations that cause

609 chromosomal rearrangements (Hardison et al. 2003, Murphy et al. 2005, Raskina et al. 2008, Molnár et

610 al. 2010, Vitte et al. 2014, Ayers-Alves et al. 2017, Li et al. 2017, Corbett-Detig et al. 2019). Given that

611 sunflowers have several genome duplications and a burst of transposable element activity in their

612 evolutionary history (Barker et al. 2008, Kawakami et al. 2011, Staton et al. 2012, Barker et al. 2016,

613 Badouin et al. 2017) it is plausible that ancestral homology or repeat content could be associated with

614 translocation propensity.

615

616 Of the above possibilities, an association between repeated translocations and centromeres would be 617 particularly compelling. Beyond the repeat content of centromeres explaining non-random mutation 618 (Kawabe et al. 2006, Sun et al. 2017, but see Lin et al. 2018, Okita et al. 2019), the position and size of 
619 centromeres on chromosomes is known to affect meiotic drive and thus the repositioning of centromeres through rearrangement could cause non-random fixation of translocations (Kaszás et al.

621 1998, Chmátal et al. 2014, Zanders et al. 2014). The relative placement of centromeres has been

622 associated with chromosome evolution in Brassica (Schranz et al. 2006) and wheat (Badaeva et al.

623 2007), and associations between meiotic drive and chromosome evolution have been found in several

624 animal taxa (Bidau and Martí 2004, Palestis et al. 2004, Molina et al. 2014, Blackmon et al. 2019). In

625 sunflower, we see some hints that centromeric repeats might be associated with repeated

626 translocation. Using the locations of the centromere-specific retrotransposon sequence, HaCEN-LINE

627 (Nagaki et al. 2015), to roughly identify the locations of centromeres in our reference, we find that

628 some rearrangement breakpoints, for example, the section of A16 with a different position in each

629 map, are close to putative centromeres (Fig S17-S20). Although a more thorough analysis of

630 centromeric repeat locations and their association with rearrangement breakpoints is required to draw

631 firm conclusions about the importance of centromeres to chromosomal evolution in sunflower, the

632 development of reference sequences for wild sunflower species is underway, which will allow those

633 and other associations to be confirmed. Further, it is time to directly test for meiotic drive in this

634 system by examining the transmission of rearrangements that affect centromeres in gametes produced

635 by plants that have heterozygous karyotypes.

637 Conclusion

638

639 Understanding the evolution of chromosomal rearrangements remains a key challenge in evolutionary

640 genetics. By developing new software to systematically detect synteny blocks and building new genetic

641 maps, we show that sunflowers exhibit rapid and non-random patterns of chromosomal evolution.

642 These data generate specific and testable hypotheses about chromosomal evolution in sunflower. We

643 believe that our work will spur additional studies of karyotypic evolution and diversity, and ultimately

644 lead to a more comprehensive understanding of the interplay between chromosomal evolution and 645 speciation. 


\section{Acknowledgments}

648 We thank Jessica Barb for providing marker sequence data, Marcy Uyenoyama for help with our 649 random walk analysis, Greg Baute for sharing hybrid seed, Chris Grassa for growing seedlings and 650 sharing scripts, and both Marco Todesco and Nadia Chaidir for help in the lab. We also thank Jenn 651 Coughlan, Andrew MacDonald, Brook Moyers, Mariano Alvarez, Dolph Schluter, Darren Irwin, Sally 652 Otto, and three anonymous reviewers for thoughtful discussions and help with earlier drafts of this 653 manuscript. This work was supported by an NSERC Postgraduate Scholarship awarded to KLO and an 654 NSERC Discovery Grant awarded to LHR (327475).

\section{Author contributions}

KLO and LHR planned the study. KLO and KS designed and built the R package syntR. KLO made genetic maps, carried out data analysis, and drafted the manuscript. All authors read, edited, and approved the final manuscript.

\section{References}

Amores A., Catchen J., Nanda I., Warren W., Walter R. et al., 2014 A RAD-tag genetic map for the platyfish (Xiphophorus maculatus) reveals mechanisms of karyotype evolution among teleost fish. Genetics 197: 625-641.

Ayres-Alves T., Cardoso A. L., Nagamachi C. Y., Sousa L. M. de, Pieczarka J. C., Noronha R. C. R., 2017 Karyotypic evolution and chromosomal organization of repetitive DNA sequences in species of Panaque, Panaqolus, and Scobinancistrus (Siluriformes and Loricariidae) from the Amazon Basin. Zebrafish 14: 251-260. 
Badouin H., Gouzy J., Grassa C. J., Murat F., Staton S. E. et al., 2017 The sunflower genome provides insights into oil metabolism, flowering and Asterid evolution. Nature 175: 1823.

Bailey J. A., Baertsch R., Kent W., Haussler D., Eichler E. E., 2004 Hotspots of mammalian chromosomal evolution. Genome Biology 5: R23-7.

Barb J. G., Bowers J. E., Renaut S., Rey J. I., Knapp S. J., et al., 2014 Chromosomal evolution and patterns of introgression in Helianthus. Genetics 197: 969-979.

Barker M. S., Kane N. C., Matvienko M., Kozik A., Michelmore R. W., et al., 2008 Multiple paleopolyploidizations during the evolution of the Compositae reveal parallel patterns of duplicate gene retention after millions of years. Molecular Biology and Evolution 25: 2445-2455.

Barker M. S., Li Z., Kidder T. I., Reardon C. R., Lai Z., Oliveira L. O., Scascitelli M., Rieseberg L. H., 2016 Most Compositae (Asteraceae) are descendants of a paleohexaploid and all share a paleotetraploid ancestor with the Calyceraceae. American Journal of Botany 103: 1203-1211.

Baute G. J., Owens G. L., Bock D. G., Rieseberg L. H., 2016 Genome-wide genotyping-by-sequencing data provide a high-resolution view of wild Helianthus diversity, genetic structure, and interspecies gene flow. American Journal of Botany 103: 2170-2177.

Berdan E. L., Kozak G. M., Ming R., Rayburn A. L., Kiehart R., Fuller R. C., 2014 Insight into genomic changes accompanying divergence: genetic linkage maps and synteny of Lucania goodei and $L$. parva reveal a Robertsonian fusion. G3: Genes| Genomes| Genetics 4: 1363-1372.

Bhutkar A., Schaeffer S. W., Russo S. M., Xu M., Smith T. F., Gelbart W. M., 2008 Chromosomal rearrangement inferred from comparisons of 12 Drosophila genomes. Genetics 179: 1657-1680.

Bidau C. J., Martí D. A., 2004 B chromosomes and Robertsonian fusions of Dichroplus pratensis (Acrididae): Intraspecific support for the centromeric drive theory. Cytogenet Genome Res 106: 347-350.

Bilton T. P., Schofield M. R., Black M. A., Chagné D., Wilcox P. L., Dodds K. G., 2018 Accounting for errors in low coverage high-throughput sequencing data when constructing genetic maps using biparental outcrossed populations. Genetics 209: 65-76.

Blackmon H., Justison J., Mayrose I., Goldberg E. E., 2019 Meiotic drive shapes rates of karyotype evolution in mammals. Evolution 73: 511-523.

Bourque G., Pevzner P. A., 2002 Genome-scale evolution: reconstructing gene orders in the ancestral species. Genome Research 12: 26-36.

Bowers J. E., Bachlava E., Brunick R. L., Rieseberg L. H., Knapp S. J., Burke J. M., 2012 Development of a 10,000 locus genetic map of the sunflower genome based on multiple crosses. G3 2: 721-729.

Broman K. W., Wu H., Sen S., Churchill G. A., 2003 R/qtl: QTL mapping in experimental crosses. 
Bioinformatics 19: 889-890.

Burke J. M., Lai Z., Salmaso M., Nakazato T., Tang S., Heesacker A., Knapp S. J., Rieseberg L. H., 2004 Comparative mapping and rapid karyotypic evolution in the genus Helianthus. Genetics 167: 449457.

Chandler J. M., Jan C. C., Beard B. H., 1986 Chromosomal differentiation among the annual Helianthus species. Systematic Botany 11: 354-371.

Chen Z., Fu B., Jiang M., Zhu B., 2009 On recovering syntenic blocks from comparative maps. J Comb Optim 18: 307-318.

Chmátal L., Gabriel S. I., Mitsainas G. P., Martínez-Vargas J., Ventura J et al., 2014 Centromere strength provides the cell biological basis for meiotic drive and karyotype evolution in mice. Current Biology 24: 2295-2300.

Choi V., Zheng C., Zhu Q., Sankoff D., 2007 Algorithms for the extraction of synteny blocks from comparative maps. In: International Workshop on Algorithms in Bioinformatics, pp. 277-288. Springer, Berlin, Heidelberg.

Corbett-Detig R. B., Said I., Calzetta M., Genetti M., McBroome J., Maurer N. W., Petrarca V., Torre della A., Besansky N. J., 2019 Fine-mapping complex inversion breakpoints and investigating somatic pairing in the Anopheles gambiae species complex using proximity-ligation sequencing. Genetics 213: 1495-1511.

da Silva W. O., Pieczarka J. C., da Costa M. J. R., Ferguson-Smith M. A., O’Brien P. C. M., MendesOliveira A. C., Rossi R. V., Nagamachi C. Y., 2019 Chromosomal phylogeny and comparative chromosome painting among Neacomys species (Rodentia, Sigmodontinae) from eastern Amazonia.BMC Evolutionary Biology 19: 1-13.

Danecek P., Auton A., Abecasis G., Albers C. A., Banks E. et al., 1000 Genomes Project Analysis Group, 2011 The variant call format and VCFtools. Bioinformatics 27: 2156-2158.

Darling A. C. E., Mau B., Blattner F. R., Perna N. T., 2004 Mauve: multiple alignment of conserved genomic sequence with rearrangements. Genome Research 14: 1394-1403.

DePristo M. A., Banks E., Poplin R., Garimella K. V., Maguire J. R. et al., 2011 A framework for variation discovery and genotyping using next-generation DNA sequencing data. Nat Genet 43: 491-501.

Doyle J., Doyle J., 1987 A rapid DNA isolation procedure for small quantities of fresh leaf tissue. Phytochem Bull 19: 11-15.

Drillon G., Carbone A., Fischer G., 2014 SynChro: A fast and easy tool to reconstruct and visualize synteny blocks along eukaryotic chromosomes. PLoS ONE 9: e92621-8. 
Dvorak J., Wang L., Zhu T., Jorgensen C. M., Deal K. R. et al., 2018 Structural variation and rates of genome evolution in the grass family seen through comparison of sequences of genomes greatly differing in size. Plant J 95: 487-503.

Ferguson-Smith M. A., Trifonov V., 2007 Mammalian karyotype evolution. Nat Rev Genet 8: 950-962.

Ferriera J. V., 1980 Introgressive hybrisization between Helanthus annuus L. and Helianthus petiolaris Nutt. Mendeliana 4: 81-93.

Fishman L., Stathos A., Beardsley P. M., Williams C. F., Hill J. P., 2013 Chromosomal rearrangements and the genetics of reproductive barriers in Mimulus (monkey flowers). Evolution 67: 2547-2560.

Flagel L. E., Blackman B. K., Fishman L., Monnahan P. J., Sweigart A., Kelly J. K., 2019 GOOGA: A platform to synthesize mapping experiments and identify genomic structural diversity (FA Feltus, Ed.). PLoS Comput Biol 15: e1006949-25.

Goel M., Sun H., Jiao W.-B., Schneeberger K., 2019 SyRI: Finding genomic rearrangements and local sequence differences from whole- genome assemblies.Genome Biology 20: 1-13.

Hackett C. A., Broadfoot L. B., 2003 Effects of genotyping errors, missing values and segregation distortion in molecular marker data on the construction of linkage maps. Heredity 90: 33-38.

Hardison R. C., Roskin K. M., Yang S., Diekhans M., Kent W. J. et al., 2003 Covariation in frequencies of substitution, deletion, transposition, and recombination during eutherian evolution. Genome Research 13: 13-26.

Heesacker A. F., Bachlava E., Brunick R. L., Burke J. M., Rieseberg L. H., Knapp S. J., 2009 Karyotypic Evolution of the Common and Silverleaf Sunflower Genomes. The Plant Genome 2: 233-14.

Heiser C. B. Jr, 1947 Hybridization between the sunflower species Helianthus annuus and H. petiolaris. Evolution 1: 249-262.

Heiser C. B. Jr, 1948 Taxonomic and Cytological Notes on the Annual Species of Helianthus. Bulletin of the Torrey Botanical Club 75: 512-515.

Heiser C. B. Jr, 1951 Hybridization in the annual sunflowers: Helianthus annuus x H. argophyllus. The American Naturalist 85: 65-72.

Heiser C. B. Jr, 1961 Morphological and cytological variation in Helianthus petiolaris with notes on related species. Evolution 15: 247-258.

Hoffmann A. A., Rieseberg L. H., 2008 Revisiting the impact of inversions in evolution: From population genetic markers to drivers of adaptive shifts and speciation? Annu. Rev. Ecol. Evol. Syst. 39: 21-42. 
Huang K., Andrew R. L., Owens G. L., Ostevik K. L., Rieseberg L. H., 2019 Multiple chromosomal inversions contribute to adaptive divergence of a dune sunflower ecotype. bioRxiv: 829622.

Huang S., Li R., Zhang Z., Li L., Gu X., et al., 2009 The genome of the cucumber, Cucumis sativus L. Nat Genet 41: 1275-1281.

Huchra J. P., Geller M. J., 1982 Groups of galaxies. I-Nearby groups. The Astrophysical Journal 257: 423-437.

Kaszás E., Genetics J. B., 1998 Meiotic transmission rates correlate with physical features of rearranged centromeres in maize. Genetics 150: 1683-1692.

Kawabe A., Hansson B., Hagenblad J., Forrest A., Charlesworth D., 2006 Centromere locations and associated chromosome rearrangements in Arabidopsis lyrata and A. thaliana. Genetics 173: 1613-1619.

Kawakami T., Dhakal P., Katterhenry A. N., Heatherington C. A., Ungerer M. C., 2011 Transposable element proliferation and genome expansion are rare in contemporary sunflower hybrid populations despite widespread transcriptional activity of LTR retrotransposons. Genome Biol Evol 3: 156-167.

King M., 1987 Chromosomal rearrangements, speciation and the theoretical approach. Heredity 59: 16.

King M., 1993 Species Evolution. Cambridge University Press.

Kirkpatrick M., Barton N., 2006 Chromosome inversions, local adaptation and speciation. Genetics 173: 419-434.

Lai Z., Nakazato T., Salmaso M., Burke J. M., Tang S. et al., 2005 Extensive chromosomal repatterning and the evolution of sterility barriers in hybrid sunflower species. Genetics 171: 291-303.

Larkin D. M., Pape G., Donthu R., Auvil L., Welge M., Lewin H. A., 2009 Breakpoint regions and homologous synteny blocks in chromosomes have different evolutionary histories. Genome Research 19: 770-777.

Latta R. G., Bekele W. A., Wight C. P., Tinker N. A., 2019 Comparative linkage mapping of diploid, tetraploid, and hexaploid Avena species suggests extensive chromosome rearrangement in ancestral diploids. Scientific Reports 9: 1-12.

Li H., 2013 Aligning sequence reads, clone sequences and assembly contigs with BWA-MEM. arXiv: 13033997.

Li H., Durbin R., 2010 Fast and accurate long-read alignment with Burrows-Wheeler transform. Bioinformatics 26: 589-595. 
803

804

805

806

807

808

809

810

811

812

813

814

815

816

817

818

819

820

821

822

823

824

825

826

827

828

829

830

831

832

833

834

835

Li S.-F., Su T., Cheng G.-Q., Wang B.-X., Li X., Deng C.-L., Gao W.-J., 2017 Chromosome evolution in connection with repetitive sequences and epigenetics in plants. Genes 8: 290-19.

Lin C.-Y., Shukla A., Grady J., Fink J., Dray E., Duijf P., 2018 Translocation breakpoints preferentially occur in euchromatin and acrocentric chromosomes. Cancers 10: 13-19.

Mandel J. R., Nambeesan S., Bowers J. E., Marek L. F., Ebert D. et al., 2013 Association mapping and the genomic consequences of selection in sunflower. PLoS Genetics 9: e1003378.

Marone D., Laidò G., Gadaleta A., Colasuonno P., Ficco D. B. M., Giancaspro A., Giove S., Panio G., Russo M. A., De Vita P., Cattivelli L., Papa R., Blanco A., Mastrangelo A. M., 2012 A high-density consensus map of A and B wheat genomes. Theor Appl Genet 125: 1619-1638.

Martinez P. A., Jacobina U. P., Fernandes R. V., Brito C., Penone C., Amado T. F., Fonseca C. R., Bidau C. J., 2016 A comparative study on karyotypic diversification rate in mammals. Heredity 118: 366373.

Mason A. S., Nelson M. N., Takahira J., Cowling W. A., Alves G. M., Chaudhuri A., Chen N., Ragu M. E., Dalton-Morgan J., Coriton O., Huteau V., Eber F., Chèvre A. M., Batley J., 2014 The fate of chromosomes and alleles in an allohexaploid Brassica population. Genetics 197: 273-283.

Mason C. M., 2018 How old are sunflowers? A molecular clock analysis of key divergences in the origin and diversification of Helianthus (Asteraceae). Int. J Plant Sci. 179: 182-191.

Matvienko M., Kozik A., Froenicke L., Lavelle D., Martineau B. et al., 2013 Consequences of normalizing transcriptomic and genomic libraries of plant genomes using a duplex-specific nuclease and tetramethylammonium chloride. PLoS ONE 8: e55913-17.

McKenna A., Hanna M., Banks E., Sivachenko A., Cibulskis K. et al., 2010 The Genome Analysis Toolkit: A MapReduce framework for analyzing next-generation DNA sequencing data. Genome Research 20: $1297-1303$.

Molina W. F., Martinez P. A., Bertollo L. A. C., Bidau C. J., 2014 Evidence for meiotic drive as an explanation for karyotype changes in fishes. Marine Genomics 15: 29-34.

Molnár I., Cifuentes M., Schneider A., Benavente E., Molnár-Láng M., 2010 Association between simple sequence repeat-rich chromosome regions and intergenomic translocation breakpoints in natural populations of allopolyploid wild wheats. Annals of Botany 107: 65-76.

Murphy W. J., Larkin D. M., Everts-van der Wind A., Bourque G., Tesler G. et al., 2005 Dynamics of mammalian chromosome evolution inferred from multispecies comparative maps. Science 309: 613-617.

Nagaki K., Tanaka K., Yamaji N., Kobayashi H., Murata M., 2015 Sunflower centromeres consist of a centromere-specific LINE and a chromosome-specific tandem repeat. Front. Plant Sci. 6: 1-12. 
Navarro A., Barton N. H., 2003 Chromosomal speciation and molecular divergence--accelerated evolution in rearranged chromosomes. Science 300: 321-324.

Nguepjop J. R., Tossim H.-A., Bell J. M., Rami J.-F., Sharma S., Courtois B., Mallikarjuna N., Sane D., Fonceka D., 2016 Evidence of genomic exchanges between homeologous chromosomes in a cross of peanut with newly synthetized allotetraploid hybrids. Front. Plant Sci. 7: 87-12.

Nicolas S. D., Mignon G. L., Eber F., Coriton O., Monod H., Clouet V., Huteau V., Lostanlen A., Delourme R., Chalhoub B., Ryder C. D., Chèvre A. M., Jenczewski E., 2007 Homeologous recombination plays a major role in chromosome rearrangements that occur during meiosis of Brassica napus haploids. Genetics 175: 487-503.

Noor M. A., Grams K. L., Bertucci L. A., Reiland J., 2001 Chromosomal inversions and the reproductive isolation of species. Proceedings of the National Academy of Sciences 98: 12084-12088.

Okita A. K., Zafar F., Su J., Weerasekara D., Kajitani T., Takahashi T. S., Kimura H., Murakami Y., Masukata H., Nakagawa T., 2019 Heterochromatin suppresses gross chromosomal rearrangements at centromeres by repressing Tfs1/TFIIS-dependent transcription. Communications Biology 2: 113.

Ostevik K. L., 2016 The ecology and genetics of adaptation and speciation in dune sunflowers.

Ouellette L. A., Reid R. W., Blanchard S. G., Brouwer C. R., 2017 LinkageMapView - Rendering High Resolution Linkage and QTL Maps. Bioinformatics 34: 306-307.

Palestis B. G., Burt A., Jones R. N., Trivers R., 2004 B chromosomes are more frequent in mammals with acrocentric karyotypes: Support for the theory of centromeric. drive. Proc. Biol. Sci. 271: 1-3.

Pevzner P., Tesler G., 2003 Genome rearrangements in mammalian evolution: lessons from human and mouse genomes. Genome Research 13: 37-45.

Poland J. A., Brown P. J., Sorrells M. E., Jannink J.-L., 2012 Development of high-density genetic maps for barley and wheat using a novel two-enzyme genotyping-by-sequencing approach. PLoS ONE 7: e32253.

Quillet M. C., Madjidian N., Griveau Y., Serieys H., Tersac M., Lorieux M., Berville A., 1995 Mapping genetic factors controlling pollen viability in an interspecific cross in Helianthus sect. Helianthus. Theor Appl Genet 91: 1195-1202.

Raduski A. R., Rieseberg L., Strasburg J., 2010 Effective population size, gene flow, and species status in a narrow endemic sunflower, Helianthus neglectus, compared to its widespread sister species, $H$. petiolaris. IJMS 11: 492-506.

Raskina O., Barber J. C., Nevo E., Belyayev A., 2008 Repetitive DNA and chromosomal rearrangements: speciation-related events in plant genomes. Cytogenet Genome Res 120: 351-357. 
Rieseberg L., 1991 Homoploid reticulate evolution in Helianthus (Asteraceae): evidence from ribosomal genes American Journal of Botany 78: 1218-1237.

Rieseberg L. H., 2001 Chromosomal rearrangements and speciation. Trends in Ecology \& Evolution 16: 351-358.

Rieseberg L. H., Linder C. R., Seiler G. J., 1995 Chromosomal and genic barriers to introgression in Helianthus. Genetics 141: 1163-1171.

Rogers C. E., Thompson T. E., Seiler G. J., 1982 Sunflowers species of the United States. National Sunflower Association.

Rohland N., Reich D., 2012 Cost-effective, high-throughput DNA sequencing libraries for multiplexed target capture. Genome Research 22: 939-946.

Rueppell O., Kuster R., Miller K., Fouks B., Rubio Correa S., Collazo J., Phaincharoen M., Tingek S., Koeniger N., 2016 A new metazoan recombination rate record and consistently high recombination rates in the honey bee genus Apis accompanied by frequent inversions but not translocations. Genome Biol Evol 8: 3653-3660.

Sambatti J. B. M., Strasburg J. L., Ortiz-Barrientos D., Baack E. J., Rieseberg L. H., 2012 Reconciling extremely strong barriers with high levels of gene exchange in annual sunflowers. Evolution $\mathbf{6 6}$ : 1459-1473.

Schlautman B., Diaz-Garcia L., Covarrubias-Pazaran G., Schlautman N., Vorsa N. et al., 2017 Comparative genetic mapping reveals synteny and collinearity between the American cranberry and diploid blueberry genomes. Molecular Breeding 38: 1-19.

Schranz M. E., Mitchell-Olds T., Lysak M. A., 2006 The ABC's of comparative genomics in the Brassicaceae: Building blocks of crucifer genomes. Trends in Plant Science 11: 535-542.

Searle J. B., 1993 Chromosomal hybrid zones in eutherian mammals. In: Hybrid zones and the evolutionary process, pp. 309-353.

Shagina I., Bogdanova E., Mamedov I., Lebedev Y., Lukyanov S., Shagin D., 2010 Normalization of genomic DNA using duplex-specific nuclease. Biotechniques 48: 455-459.

Sinha A. U., Meller J., 2007 Cinteny: Flexible analysis and visualization of synteny and genome rearrangements in multiple organisms. BMC Bioinformatics 8: 82-9.

Soderlund C., Bomhoff M., Nelson W. M., 2011 SyMAP v3.4: A turnkey synteny system with application to plant genomes. Nucleic Acids Research 39: e68-e68.

Stathos A., Fishman L., 2014 Chromosomal rearrangements directly cause underdominant F1 pollen sterility in Mimulus lewisii-Mimulus cardinalis hybrids. Evolution 68: 3109-3119. 
903

Staton S. E., Bakken B. H., Blackman B. K., Chapman M. A., Kane N. C. et al., 2012 The sunflower (Helianthus annuus L.) genome reflects a recent history of biased accumulation of transposable elements. The Plant Journal 72: 142-153.

Stephens J. D., Rogers W. L., Mason C. M., Donovan L. A., Malmberg R. L., 2015 Species tree estimation of diploid Helianthus (Asteraceae) using target enrichment. American Journal of Botany 102: 910920 .

Strasburg J., Rieseberg L., 2008 Molecular demographic history of the annual sunflowers Helianthus annuus and $H$. petiolaris-Large effective population sizes and rates of long-term gene flow. Evolution 62: 1936-1950.

Sun S., Yadav V., Billmyre R. B., Cuomo C. A., Nowrousian M., Wang L., Souciet J.-L., Boekhout T., Porcel B., Wincker P., Granek J. A., Sanyal K., Heitman J., 2017 Fungal genome and mating system transitions facilitated by chromosomal translocations involving intercentromeric recombination. PLoS Biol 15: e2002527-31.

Tang S., Yu J. K., Slabaugh M. B., Shintani D. K., Knapp S. J., 2002 Simple sequence repeat map of the sunflower genome. TAG Theoretical and Applied Genetics 105: 1124-1136.

Taylor J., Butler D., 2017 RPackage ASMap: Efficient Genetic Linkage Map Construction and Diagnosis. J. Stat. Soft. 79: 1-29.

Tennessen J. A., Govindarajulu R., Ashman T.-L., Liston A., 2014 Evolutionary origins and dynamics of octoploid strawberry subgenomes revealed by dense targeted capture linkage maps. Genome Biol Evol 6: 3295-3313.

Todesco M., Owens G. L., Bercovich N., Légaré J.-S., Soudi S., Burge D. O., Huang K., Ostevik K. L., Drummond E. B. M., Imerovski I., Lande K., Pascual M. A., Cheung W., Staton S. E., Muños S., Nielsen R., Donovan L. A., Burke J. M., Yeaman S., Rieseberg L. H., 2019 Massive haplotypes underlie ecotypic differentiation in sunflowers. bioRxiv: 790279.

Trickett A. J., Butlin R. K., 1994 Recombination suppressors and the evolution of new species. Heredity 73: 339-345.

Van der Auwera G. A., Carneiro M. O., Hartl C., Poplin R., del Angel G. et al., 2013 From fastQ data to high-confidence variant calls: The genome analysis toolkit best practices pipeline. Current Protocols in Bioinformatics 43: 11.10.1-33.

Vitte C., Fustier M. A., Alix K., Tenaillon M. I., 2014 The bright side of transposons in crop evolution. Briefings in Functional Genomics 13: 276-295.

Vogel J. P., Garvin D. F., Mockler T. C., Schmutz J., Rokhsar D. et al., 2010 Genome sequencing and analysis of the model grass Brachypodium distachyon. Nature 463: 763-768.

Weiss-Schneeweiss H., Schneeweiss G. M., 2012 Karyotype Diversity and Evolutionary Trends in 
Angiosperms. In: Plant Genome Diversity Volume 2 pp. 209-230.

938 Whelan E. D., 1979 Interspecific hybrids between Helianthus petiolaris Nutt. and H. annuus L.: Effect of backcrossing on meiosis. Euphytica 28: 297-308.

940 White M. J. D., 1973 Animal Cytology and Evolution. Cambridge University Press, London.

941 White M. J. D., 1978 Modes of Speciation. W. H. Freeman \& Co., San Francisco.

942 Wu F., Tanksley S. D., 2010 Chromosomal evolution in the plant family Solanaceae. BMC Genomics 11: 943182.

944 Yeaman S., 2013 Genomic rearrangements and the evolution of clusters of locally adaptive loci. 945 Proceedings of the National Academy of Sciences 110: E1743-E1751.

946 Yeaman S., Whitlock M., 2011 The genetic architecture of adaptation under migration-selection 947 balance. Evolution 65: 1897-1911.

948 Yogeeswaran K., Frary A., York T. L., Amenta A., Lesser A. H., Nasrallah J. B., Tanksley S. D., Nasrallah M. 949 E., 2005 Comparative genome analyses of Arabidopsis spp.: inferring chromosomal rearrangement events in the evolutionary history of A. thaliana. Genome Research 15: 505-515.

954 Zhulidov P. A., 2004 Simple cDNA normalization using kamchatka crab duplex-specific nuclease. Nucleic 955 Acids Research 32: 37e-37. 